\title{
Análisis cuantitativo por difracción de rayos $X$ de cuarzo-alfa en una matriz de cemento portland anhidro con equipos Philips modelo PW-1710 y PW-1700
}

SAGRERA MORENO, J. L., Dr. en Ciencias Quimicas y Ledo. en Ciencias de la Información IETCC/CSIC

\section{$R E S U M E N$}

En el presente articulo se explican los pasos necesarios para realizar un análisis cuantitativo de cuarzo-alfa en una matriz de cemento portland anhidro, mediante la simple adición de una pequeña cantidad de CORINDON a la muestra y la aplicación de una sencilla fórmula matemática (1) que directamente proporciona el porcentaje del compuesto cristalino que se quiere determinar. Con este método se elimina la laboriosa preparación de muestras con patrón interno, necesarias para obtener una curva de calibrado. Además se elimina la necesidad de poseer muestra pura del componente que se quiere analizar. El método es sencillo y rápido, dando una precisión aceptable en las determinaciones cuantitativas de compuestos cristalinos.

\section{$S U M M A R Y$}

This article, explains the necessary steps to obtained a quartz-low quantitative analysis in an anhydrous portland cement matrix, by means of a simple addition of a little quantity of Corindon to the sample and applying a simple mathematical formula which gives directly the crystalline compound percentage which determination is required. Whith this method the labour of preparation samples with internal standard, necessary to obtain a straight line, is eliminated. The need of having a pure sample of the compound which is to be analyzed is also excluded. The method is simple and rapid, offering an acceptable precision in the quantitative crystalline compounds determination.

\section{Parte 1. ${ }^{\mathrm{a}}$ : modelo PW-1710}

\section{PREPARACION DE LA MUESTRA}

Se pesan exactamente 2,00 gramos de cemento portland anhidro, 0,20 gramos de cuarzo-alfa (procedente de Segovia) con un tamaño de grano inferior a 4.900 mallas y 0,40 gramos de CORINDON SINTETICO marca JANSSEN ( $99 \%$ de $\mathrm{Al}_{2} \mathrm{O}_{3}$ ). El conjunto de los tres componentes se homogeiniza en un molino vibratorio marca SPECAMILL de la firma SPECAC (Londres) durante cinco minutos, utilizando un recipiente cilindrico de CELOTEX de 48 milimetros de altura y 13 milimetros de diámetro interno, tapado en ambas bases con tapones de goma virgen, dentro del cual se introduce la muestra y 3 bolas de acero de 8 milímetros de diámetro. Extraida la muestra del cilindro de CELOTEX, se prensa sobre el portamuestras de difracción de rayos $\mathrm{X}$, utilizando una prensa hidráulica marca HERZOG tipo HDIFP mediante la técnica especificada en (2).

La muestra preparada, se denomina CURSO4 y las cantidades en tanto por ciento son:

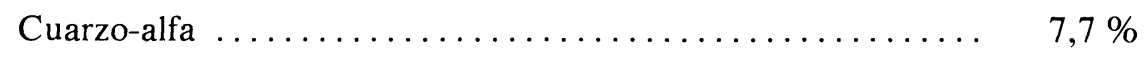

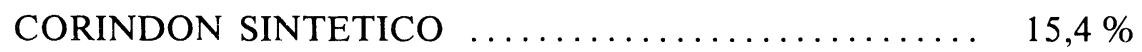


$\mathrm{C}=$ LSS (colocación de la muestra en posición de medida).

$\mathrm{C}=$ SAN 26,75 (ángulo teórico de recuento del cuarzo).

$\mathrm{C}=$ MXN 10 (número de puntos de medida a cada lado del valor teórico $2 \theta$ ).

$\mathrm{C}=\operatorname{MXS}$ 0,005 (tamaño de cada paso del goniómetro).

$\mathrm{C}=$ MXT 10 (tiempo de recuento de cada paso).

$\mathrm{C}=\operatorname{MAX}$ (orden de comienzo del trabajo para búsqueda del valor $2 \theta$ máximo).

26,750 (valor experimental del máximo $2 \theta$ ).

1.482 (número de cuentas/seg. en $2 \theta$ máximo).

Los valores dados a MXN y MXS, deben ser función de la forma que tenga el pico considerado, y que para nuestro caso puede comprobarse que $10 \times 0,005=0,05$, valor que añadido o sustraido a $2 \theta=26,75$ indica los valores extremos de recuento $2 \theta$, es decir $26,75 \pm 0,05=26,70$ y 26,80 , valores de $2 \theta$ donde no hay interferencias en el pico máximo del cuarzo.

\section{RESULTADOS OBTENIDOS}

En la tabla 1 pueden verse los programas completos para recuento en el pico máximo del CUARZO-alfa y del CORINDON SINTETICO. Los resultados obtenidos son:

\begin{tabular}{|c|c|c|}
\hline COMPUESTO & PICO MAXIMO $2 \theta$ & CUENTAS/seg \\
\hline CUARZO-alfa & 26,750 & 1.482 \\
\hline CORINDON SIN & 57,575 & 873 \\
\hline
\end{tabular}

Estos valores de recuentos, se llevan a la fórmula para el cálculo del tanto por ciento del compuesto cristalino problema, que forma parte de la muestra analizada

$$
\mathrm{Xi}=\frac{\mathrm{Xc}}{\mathrm{Ki}} \cdot \frac{\mathrm{Ii}}{\mathrm{Ic}}
$$

donde:

$\mathrm{Xi}=\%$ CUARZO alfa en muestra.

$\mathrm{Xc}=\%$ CORINDON SIN. en muesta.

Ii $=$ Intensidad en cuentas/seg del pico máximo del CUARZO.

Ic $=$ Intensidad en cuentas/seg del pico máximo del CORINDON.

$\mathrm{Ki}=\mathrm{Ia} / \mathrm{Ib}$.

donde:

Ia $=$ Intensidad en cuentas/seg. del pico máximo del cuarzo en una muestra $100 \%$ CUARZO.

$\mathrm{Ib}=$ Intensidad en cuentas/seg. del pico máximo del CORINDON en una muestra $100 \%$ CORINDON.

En muchos casos el valor de Ki puede encontrarse en el libro POWER DIFFRACTION FILE INORGANIC PHASES (1982) publicado por el J.C.P.D.S. 
En nuestro caso dicho valor es 3,60

$$
\% \text { CUARZO }=\frac{15,4}{3,60} \cdot \frac{1.482}{873}=7,3 \%
$$

resultando acorde con el porcentaje de cuarzo que la muestra tiene $(7,7 \%)$.

La falta de exactitud del método, puede englobar errores de homogeneización de muestra y del valor Ki. Valor éste, que debe ser obtenido experimentalmente cuando se tenga muestra pura del compuesto cristalino que se desee determinar su contenido en la muestra analizada.

\section{Parte 2.': modelo PW-170}

\section{PREPARACION DE LA MUESTRA}

La muestra esta constituida por 2,00 gramos de cemento portland anhidro, 0,20 gramos de cuarzo-alfa (procedente de Segovia) con un tamaño de grano inferior a 4.900 mallas y 0,40 gramos de corindón sintético, marca Janssen $\left(99 \% \mathrm{Al}_{2} \mathrm{O}_{3}\right)$; lo que supone un contenido en la muestra total de $7,7 \%$ de cuarzo-alfa.

Los tres componentes se homogeinizan en un molino Specamill (de la firma Specac-Londres) durante cinco minutos, utilizando un recipiente cilindrico de Celotex y tres bolas de acero de 8 milímetros de diámetro como elementos mezcladores. La muestra así preparada se ha llamado CURSO4.

\section{DESCRIPCION DEL EQUIPO}

Se ha empleado un equipo automático de difracción de rayos $\mathrm{X}$ compuesto de las siguientes unidades:

- Generador de alta tensión de $4 \mathrm{~kW}, \mathrm{PW}-1730$.

- Rendija automática de divergencia, PW-1386/50.

- Monocromador de grafito, PW-1743/00.

- Portamuestras automático para 42 muestras, PW-1780.

- Unidad de refrigeración automática, DM-6000.

- Software analítico Philips APD-1700 (V.s.2.0).

- Miniordenador Digital modelo PDP 11/24 de 256 Kbytes.

- Teleimpresora Digital modelo LA-120.

- Terminal gráfico Hewlett Packard modelo 2648 A.

- Impresora gráfica Hewlett Packard modelo 2631 G.

- Prensa semiautomática Herzog modelo HDIFP.

- Portamuestras modificados por el I.E.T.c.c. (diámetro $=20 \mathrm{~mm}$ ). 


\section{ANALISIS CUALITATIVO DE LAS FASES MAYORITARIAS}

Con el objeto de estudiar los picos característicos, para sobre ellos efectuar los recuentos de impulsos que producen la intensidad (resultados) que se aplican a la fórmula matemática, se ha realizado un análisis cualitativo de las fases cristalinas mayoritarias de la muestra CURSO4. Las condiciones de trabajo son:

- Anodo de cobre trabajando a $40 \mathrm{kV}$ y $50 \mathrm{~mA}$.

- Tamaño de paso del goniómetro 0,02 grados.

- Tiempo de recuento en cada paso 0,80 segundos.

- Recorrido del goniómetro desde $2 \theta=5^{\circ}$ hasta $2 \theta=75^{\circ}$.

- Niveles del discriminador de altura de impulsos, máximo 70 voltios mínimo 35 voltios.

- Portamuestras rotatorio.

El difractograma obtenido, proporciona un listado de 80 picos cristalinos de los cuales y fijando la condición de $\mathrm{I} / \mathrm{Imax}$ mayor que $25 \%$, se han listado los 10 picos cristalinos de la Tabla 1. En dicha tabla puede comprobarse que los siete primeros picos, con valores de ángulo 26,7600 a 43,4325 tienen doble tipo de pico Alfa1 y Alfa2 y los tres picos restantes números 8,9 y 10 con tipo único de pico Alfa1.

La identificación cualitativa de los compuestos cristalinos mayoritarios presentes en la muestra, se hace mediante el software APD-1700 por comparación de los picos de la muestra, con los picos de las fichas de referencia codificadas en un disco del miniordenador, utilizando los valores de los picos del tipo Alfa1, por ello y aplicando un programa de software, se eliminan mediante dicho programa todos los picos del tipo Alfa2, obteniéndose un nuevo listado de picos en la Tabla 2, donde puede comprobarse la eliminación de Alfa2 y la correspondiente variación de los valores angulares de los picos listados. La variación de los valores de los picos, arrastra la variación de todos los parámetros obtenidos en la Tabla 1, pasando de los 80 picos cristalinos a los 70 picos, como resultado de esta condición de eliminación.

Los compuestos cristalinos mayoritarios identificados en el análisis cualitativo, aplicando el programa Sandman del software son:

- cuarzo-alfa (Quartz low ficha 5-490),

- corindón sintético (Corundum sin. ficha 10-173), y

- silicato tricálcico $\left(\mathrm{Ca}_{3} \mathrm{SiO}_{5}\right.$ ficha 11-593),

cuyos diagramas de barras pueden verse en la figura 1. Dichos diagramas de barras comparados con el diagrama de barras de la muestra analizada (File: CURSO4.DI) parte superior de la figura 1, demuestran por la coincidencia entre barras (picos) de la muestra y barras (picos) de los compuestos cristalinos mayoritarios, los identificados en el análisis cualitativo. De la observación de los diagramas de barras del Cuarzo-alfa y del Corindón sintético puede decirse que el pico máximo de intensidad (altura) para cada uno de ellos, se encuentra en los ángulos $2 \theta=26,75$ y $2 \theta=43,44$ respectivamente; no ocurriendo así en el listado de picos de la Tabla 2, donde puede apreciarse que si bien para el cuarzo-alfa, su pico máximo se mantiene en el ángulo $2 \theta=26,75$, en el caso del corindón sintético existe un pico de mayor intensidad (I/Imax) para valor $2 \theta=57,56$ que el anteriormente citado de $2 \theta=43,44$, este efecto es debido al uso de rendija automática de divergencia, que hace variar los valores de las intensidades relativas de los picos considerados. Las fichas de referencia $5-490$ y $10-173$ pertenecientes al J.C.P.D.S. (año 1982) al no estar corregidas para rendija automática de divergencia, presentan en sus diagramas de barras esta anomalia, que no sucede en el listado de picos de la Tabla 2. 


$$
\frac{\text { Angulos }}{43,4425}
$$

I/Imax Tabla 1

29,15

46,30

Por lo tanto para nuestro estudio cuantitativo aplicando la fórmula matemática de Chung, utilizaremos los picos $2 \theta=26,75$ para el cuarzo-alfa y $2 \theta=57,56$ para el Corindón sintético. Dichos picos tomados para el estudio, no presentan interferencias según puede comprobarse en las figuras 2 y 3 respectivamente, donde utilizando el programa Plot del software se han obtenido los diagramas parciales de la muestra analizada.

Una vez conocidos los picos máximos reales de los compuestos, necesarios para el cálculo automático de intensidades, se procede al cálculo de los mismos, utilizando los programas del software Phases y Search for maximum. La Phase número 10 se ha empleado para el cuarzoalfa y la Phase número 20 para el Corindón sintético; en la Tabla 3 pueden verse los parámetros fijados a cada una de estas fases, necesarios para el cálculo automático de las intensidades:

\section{Phase $.^{\circ} \mathbf{1 0}=$ Cuarzo-alfa}

Peak angle $=26,750$ (valor teórico del ángulo $2 \theta$ ).

Peak time $=10$ ( iiempo en segundos del recuento sobre el pico experimental calculado).

$\mathrm{nr} \quad=10$ (número de pasos del goniómetro a cada lado del pico teórico del ángulo $2 \theta$ ).

stpsz $\quad=0,05$ (anchura de cada paso de goniómetro).

time $\quad=10$ (tiempo en segundos de recuento en cada paso de goniómetro).

T A B LA 1

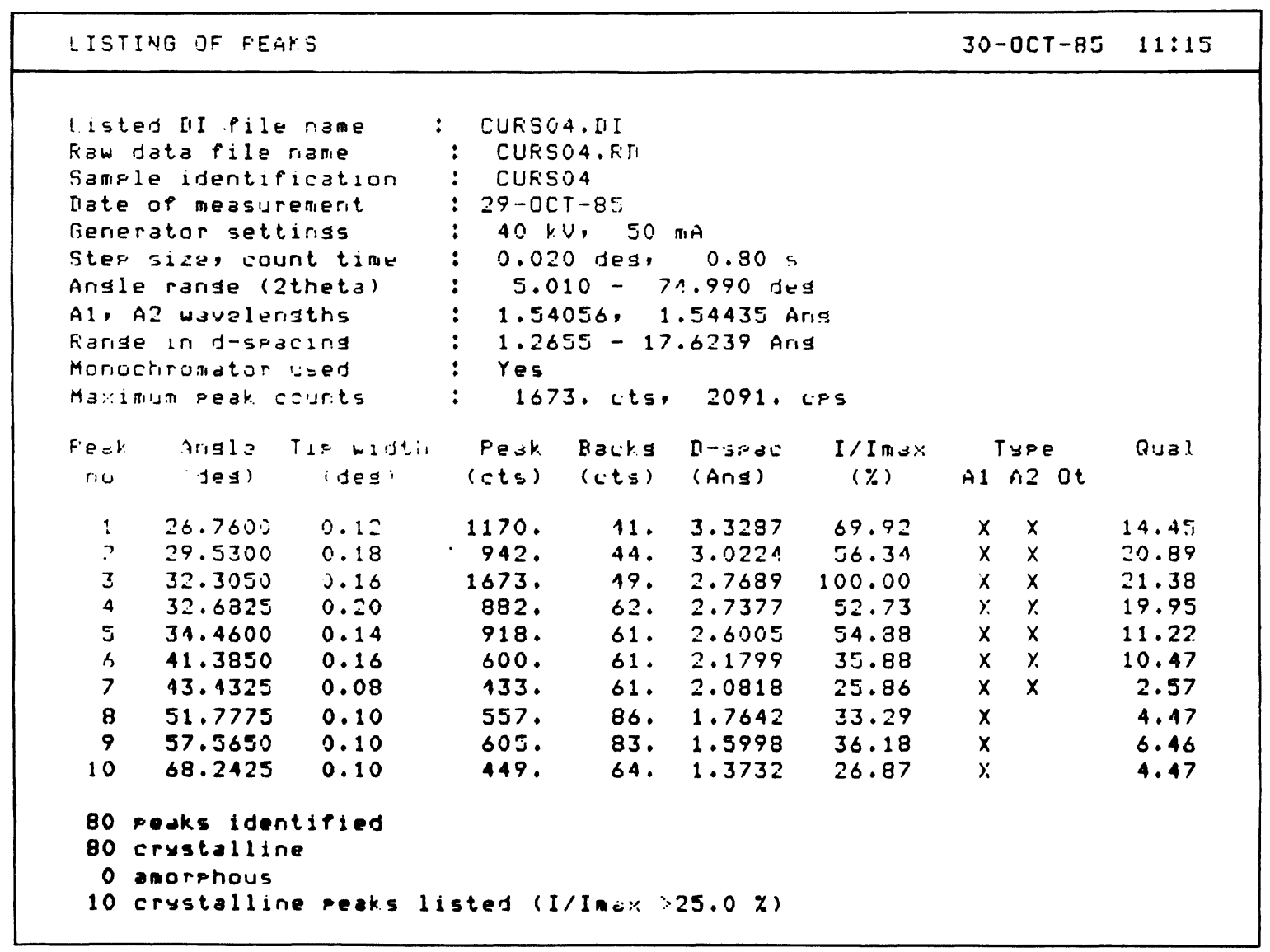




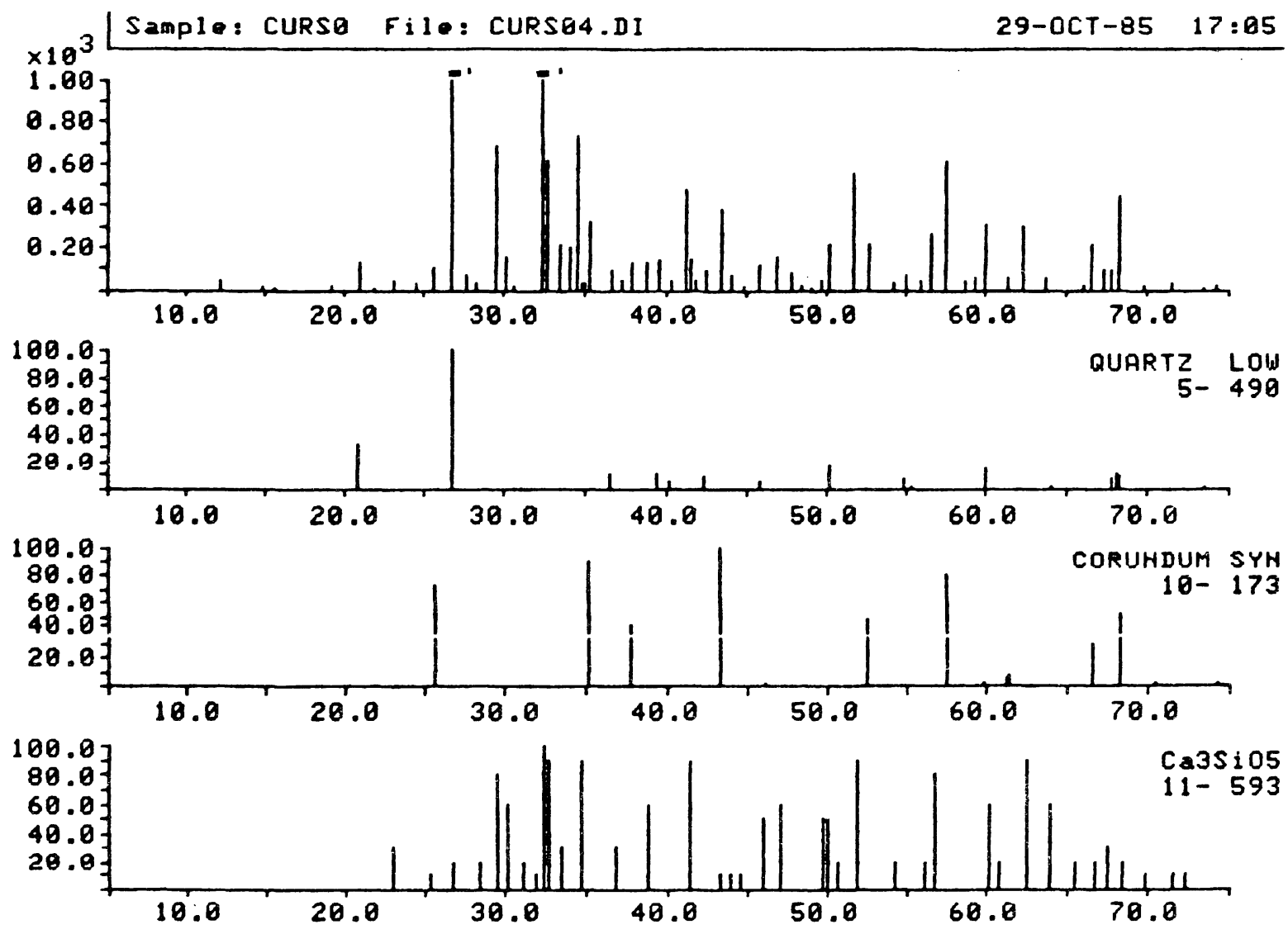

Fig. 1

TABLA 2

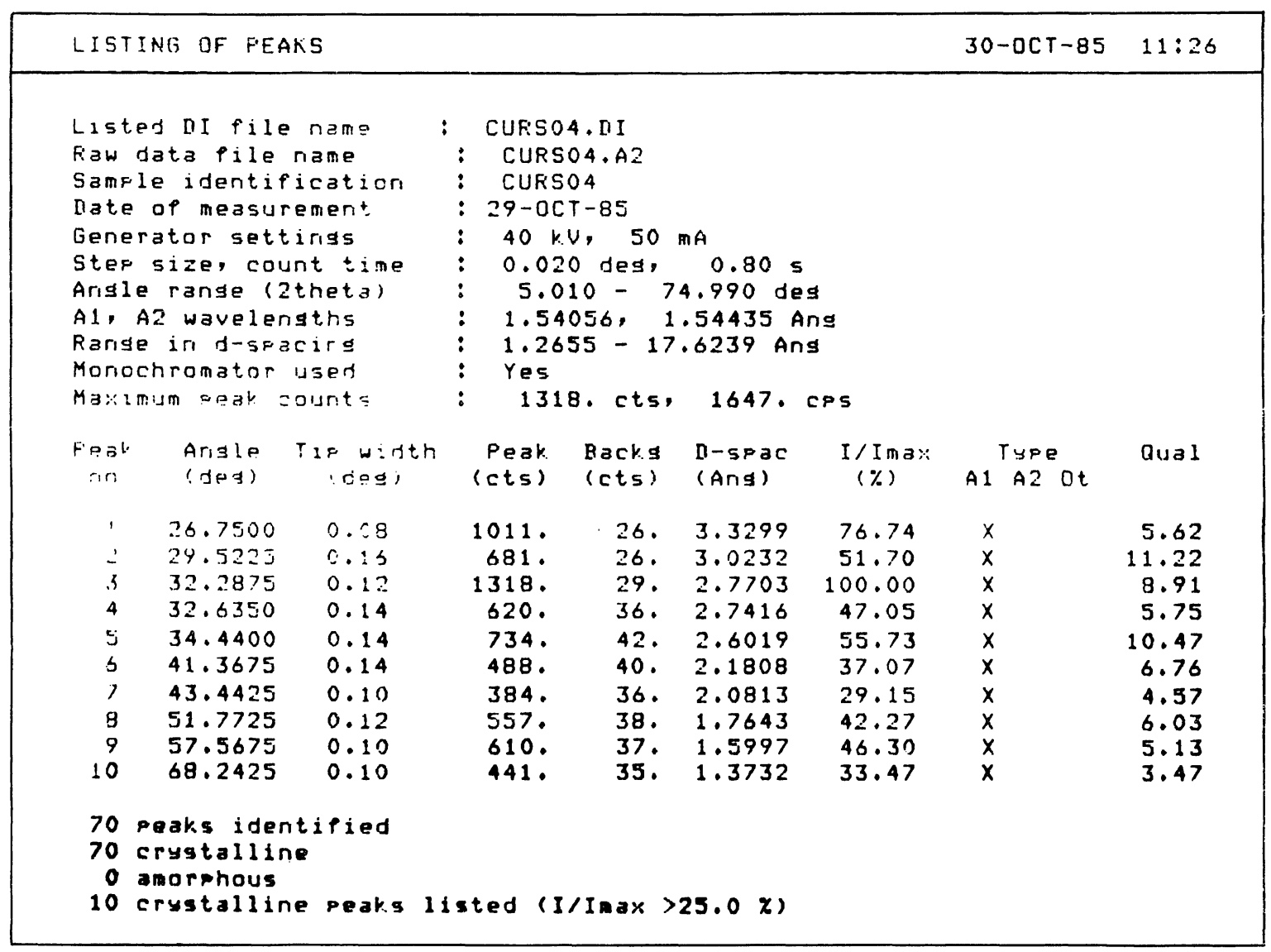




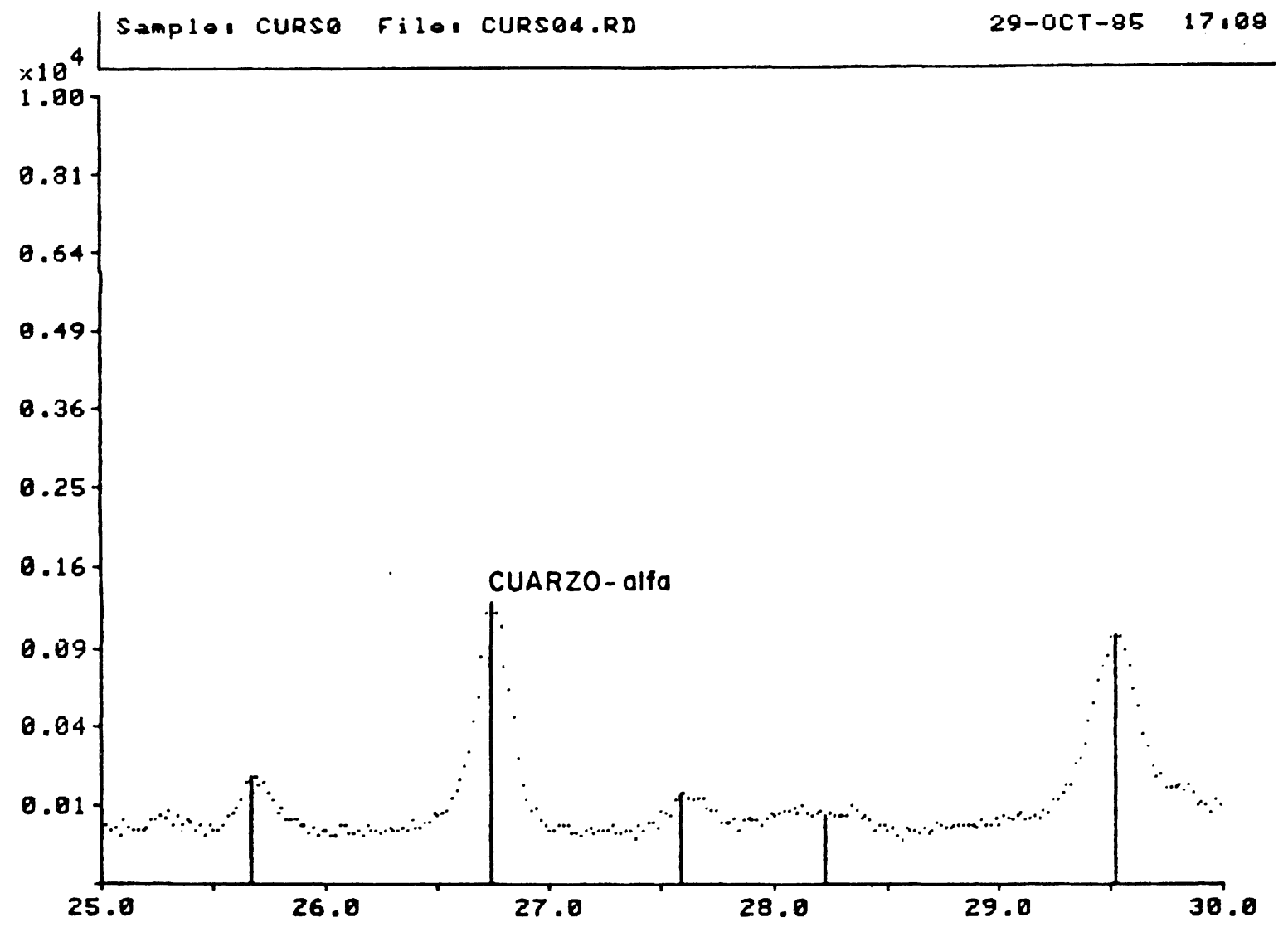

Fig. 2

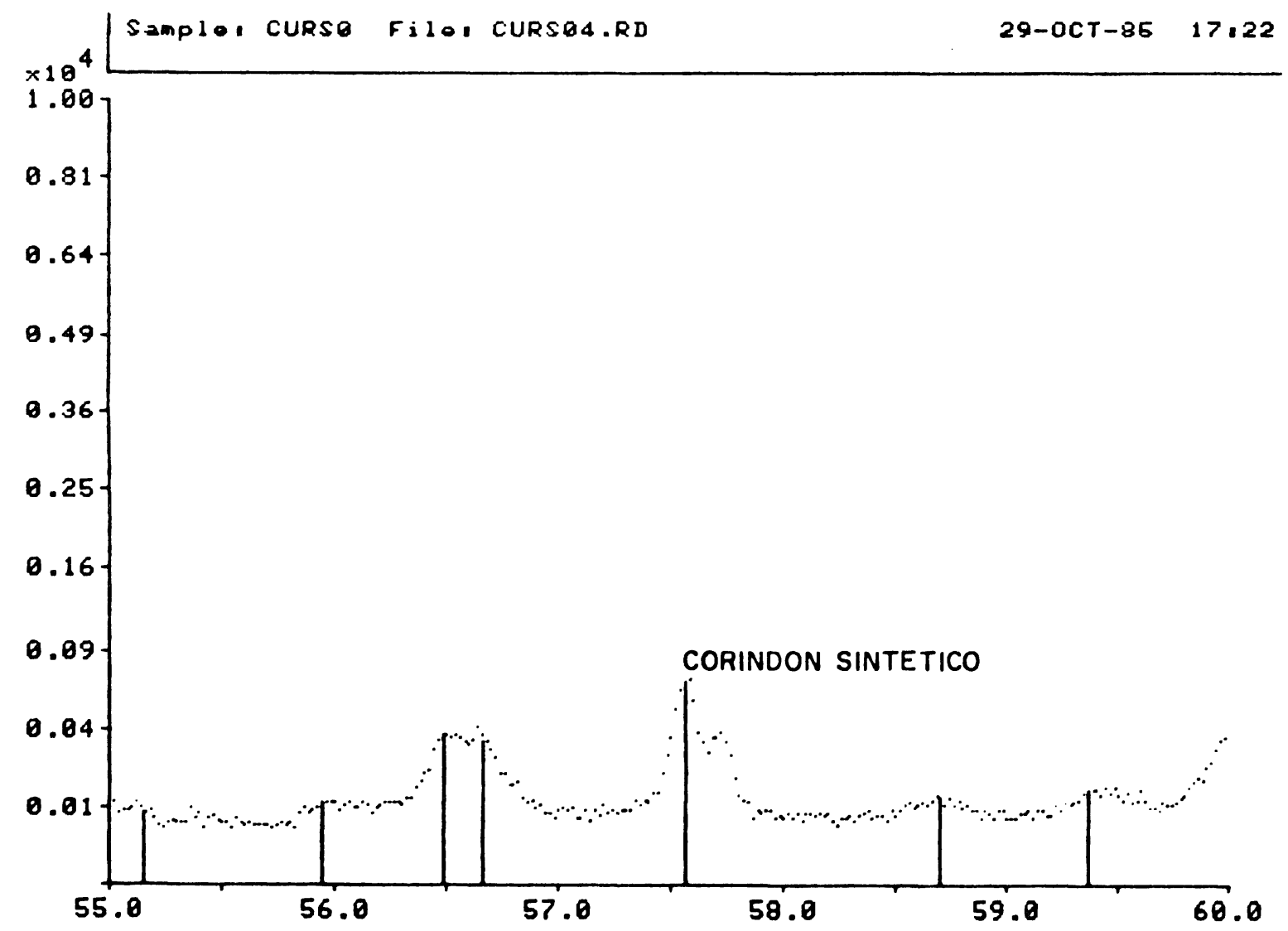

Fig. 3 
T A B LA 3

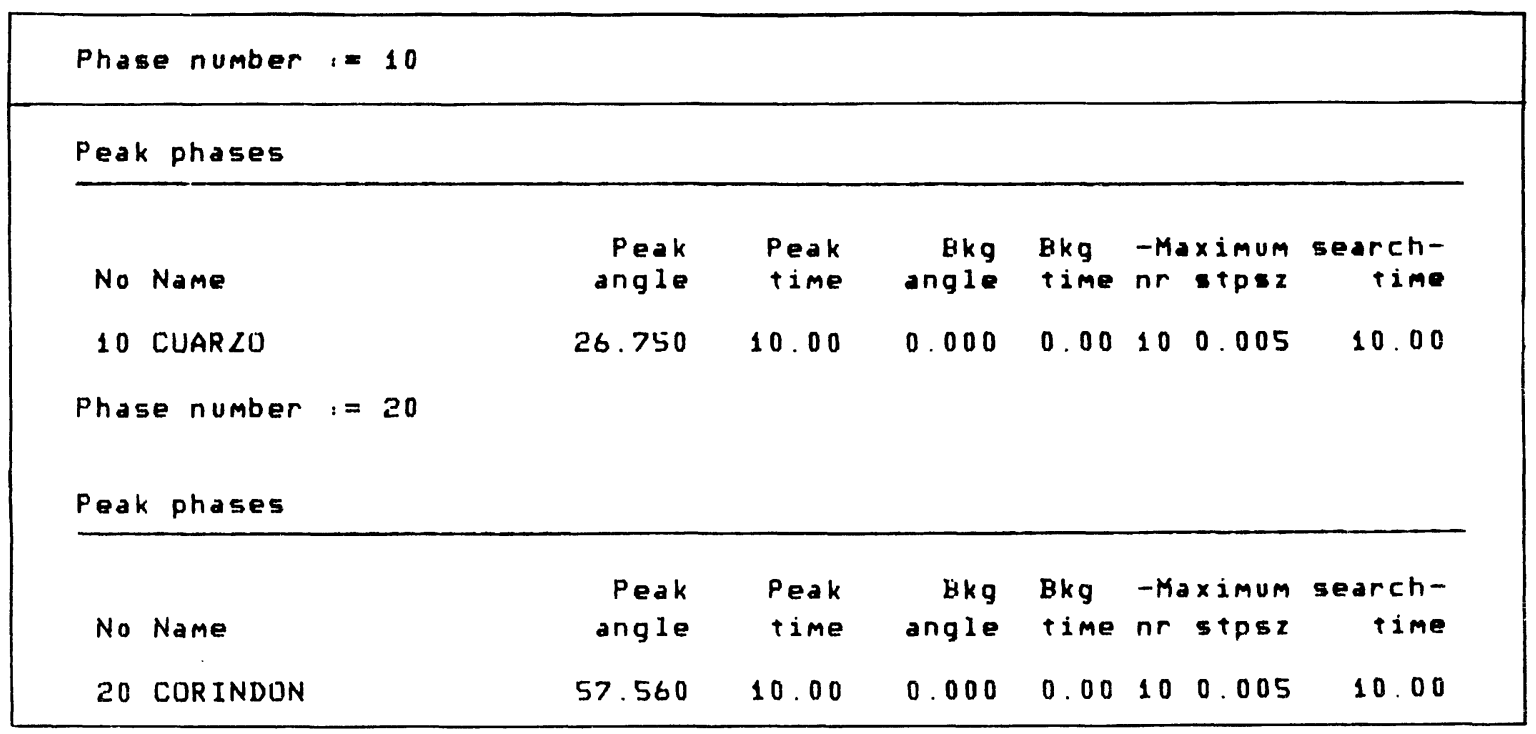

Phase n.o $20=$ Corindón sintético

Peak angle $=57,560$.

Peak time $=10$.

$\mathrm{nr} \quad=10$.

stpsz $\quad=0,05$.

time $\quad=10$.

Los parámetros "nr" y "stpsz" para cada una de las Phases, se han tomado en función de los picos considerados en las figuras 2 y 3 , donde no aparecen picos próximos que interfieran a los tomados para el estudio; los parámetros de tiempo, se han tomado en función a una buena estadistica de recuento. Mediante estas condiciones se realiza en el miniordenador del equipo, el cálculo del valor EXPERIMENTAL de la posición del pico donde se realizará, automáticamente, la medida de la intensidad del recuento. En la Tabla 4 pueden verse los resultados finales obtenidos:

- Angulo del pico máximo de la fase $10=26,745$.

- Angulo del pico máximo de la fase $20=57,575$.

TABLA 4

\begin{tabular}{|c|c|c|c|c|c|c|}
\hline \multicolumn{5}{|c|}{ QUANTITATIUE ANALYSIS } & \multicolumn{2}{|c|}{$29-0 C T-85$} \\
\hline \multicolumn{3}{|c|}{$\begin{array}{l}\text { Samle identification: } \\
\text { Quantitative data file: }\end{array}$} & \multicolumn{2}{|l|}{$\begin{array}{l}\text { CURSO4 } \\
\text { IIL:CURSO4.QI }\end{array}$} & \multirow[b]{2}{*}{$\begin{array}{l}\text { Backsround } \\
\text { correction }\end{array}$} & \multirow[b]{2}{*}{$\begin{array}{l}\text { Correcter } \\
\text { iriterisit: }\end{array}$} \\
\hline $\begin{array}{l}\text { Phase } \\
\text { number }\end{array}$ & $\begin{array}{l}\text { Phase } \\
\text { tyoe }\end{array}$ & $\begin{array}{l}\text { Phase } \\
\text { angle }\end{array}$ & $\begin{array}{l}\text { Gross } \\
\text { iriterisity }\end{array}$ & $\begin{array}{l}\text { Backsround } \\
\text { iriterisity }\end{array}$ & & \\
\hline $\begin{array}{l}10 \\
20\end{array}$ & $\begin{array}{l}\text { Peak. } \\
\text { Peak }\end{array}$ & $\begin{array}{l}26.745 \\
57.575\end{array}$ & $\begin{array}{r}1481.1 \\
857.5\end{array}$ & $\begin{array}{l}0.0 \\
0.0\end{array}$ & $\begin{array}{l}0.0 \\
0.0\end{array}$ & $\begin{array}{r}1481.1 \\
857.5\end{array}$ \\
\hline
\end{tabular}


- Intensidad corregida de la fase $10=1.481,1$ cuentas/segundo.

- Intensidad corregida de la fase $20=857,5$ cuentas/segundo.

\section{APLICACION DE LA FORMULA MATEMATICA DE CHUNG}

La fórmula a la cual se aplican directamente los resultados obtenidos es la siguiente:

donde:

$$
\mathrm{Xi}=\frac{\mathrm{Xc}}{\mathrm{Ki}} \cdot \frac{\mathrm{Ii}}{\mathrm{Ic}}
$$

$\mathrm{Xi}=\%$ Cuarzo-alfa en la muestra CURSO4.

$\mathrm{Xc}=\%$ Corindón sintético en la muestra CURSO4.

Ii = Intensidad del pico máximo del cuarzo-alfa.

Ic $=$ Intensidad del pico máximo del corindón sintético.

$\mathrm{Ki}=\mathrm{Ia} / \mathrm{Ib}$.

donde:

Ia $=$ Intensidad del pico máximo del cuarzo-alfa en una muestra $100 \%$ cuarzo-alfa.

$\mathrm{Ib}=$ Intensidad del pico máximo del corindón sintético en una muestra $100 \%$ corindón sintético.

Sustituyendo en la fórmula tenemos:

$$
\% \text { cuarzo-alfa }=\frac{15,4}{3,6} \cdot \frac{1.481,1}{857,5}=7,4 \%
$$

Resultado que difiere poco del porcentaje de cuarzo-alfa en la muestra analizada (7,7 \%).

El valor de Ki puede tomarse de las tablas Powder Diffracción File Inorganic Phases publicado por el J.C.P.D.S. (1982) y cuyo valor es 3,6; aunque para eliminar los errores instrumentales del equipo, este valor debe calcularse experimentalmente mediante muestras puras de cuarzo-alfa y corindón sintético.

\section{B I B L I O G R A F I A (Parte 1. $\left.{ }^{a}\right)$}

(1) FRANK H. CHUNG.: Quantitative Interpretation of X-ray diffraction Patterns of mixtures. I. Matrix. Flushing Method for Quantitative Multicomponents Analysis. J. Appl. Cryst. (1974) 7.519-525.

(2) J. L. SAGRERA: Preparación de muestras para análisis por difracción de rayos X con el equipo Philips PW-1700. Materiales de Construcción, Vol. 34. n. ${ }^{0}$ 195, julio/agosto/septiembre 1984.

\section{B I B L I O G R A F I A (Parte 2..$\left.^{\mathrm{a}}\right)$}

(1) FRANK H. CHUNG (1974): Quantitative Interpretation of X-ray Diffraction Patterns of Mixtures. I. Matrix-Flushing Method for Quantitative Multicomponent Analysis. J. Appl. Cryst. 7.519-525.

(2) SAGRERA-MORENO, J. L. (1985): Aplicaciones del equipo automático de difracción de polvo marca Philips modelo PW-1700 instalado en el Instituto "Eduardo Torroja" de la construcción y del cemento. Materiales de Construcción. Vol. 34. n. ${ }^{\circ} 196$, octubre/noviembre/diciembre 1984.

(3) SAGRERA-MORENO, J. L. (1985): Preparación de muestras para análisis por difracción de rayos X con el equipo Philips PW-1700. Materiales de Construcción. Vol. 34. n.o 195, julio/agosto/scptiembre 1984. 